\title{
Supervision quartets
}

\section{John Launer}

Lovers of classical music may disagree about many things, but if you ask them to name the ideal musical form they will almost all say it is the string quartet. There are many reasons why the quartet has this revered place in the musical repertoire. The intimacy of the form allows composers to express profound ideas and emotions that are more diluted in their symphonies, concertos and operas. Probably because of this, a number of great composers have turned to it to produce masterpieces, particularly in later life. These include Mozart, Haydn, Beethoven, Schubert, Dvořak, Bartok, Shostakovitch, Britten and Tippett. The sound of a quartet usually demands a small auditorium, leading to a closer sense of connection between the players and their listeners. The harmonies and counterpoint of a quartet performance may also recall our most intense human conversations. It may be no coincidence that the four string instruments playing together seem to resemble the archetypal nuclear family of mother, daughter, son and father. Indeed, many famous compositions appear to evoke this metaphor intentionally, in the way that the separate musical voices put forward their statements and respond to each other.

It may also be no coincidence that in everyday human encounters it is probably a maximum of four people-five at most-who can all pay attention to each others' views at the same time. This appears to be a constant across many different contexts and cultures. Beyond that number, a conversation will easily veer off in random directions, with two or more subgroups starting to talk to each other independently, sometimes with the formation of alliances, factions and rivalries. When four or five people are gathered together, there is conversational synergy. In a meeting of six or more people, you need a chair or facilitator, rather like an orchestral conductor.

Although the similarity between musical and conversational quartets has struck me only recently, I have some experience of both. One of my close

Correspondence to Dr John Launer, London Deanery, Stewart House, London WC1B 5DN, UK;

jlauner@londondeanery.ac.uk friends at university was a brilliant cello player who founded a string quartet. I have followed his ensemble from their early days performing in draughty suburban halls, to admiring them now at major venues in central London that are fully booked a few months in advance. Meanwhile, in my own career, I have been part of a team of educators who have introduced a kind of conversational counterpart into postgraduate medical education: the four-person peer supervision group, or 'supervision quartet'.

\section{VARIETIES OF SUPERVISION}

Peer supervision on clinical cases is not as well established in medicine as it is in some other fields such as clinical psychology. When it does occur, it generally takes two forms. The commonest is one-to-one supervision, when a doctor simply talks through a challenging case with a colleague-either on a regular, formal basis or simply when the need arises. The other well-known type of peer supervision is the case discussion group; this has a variety of different guises, including case review meetings and Balint groups. Supervision quartets combine the advantages of both these formats. They offer the focus of having a single colleague asking questions and offering guidance, while also having two other people present to listen to the conversation and offer their own reflections.

The idea of the supervision quartetfour colleagues joining together to discuss cases together-originated in general practice. ${ }^{1}$ However, since creating the method, we have used it with doctors in other specialities as diverse as anaesthetics, pathology surgery and psychiatry, and it seems to work equally well there. While it has not yet achieved the international fame of my musical friends, we have so far managed to demonstrate the method in a dozen or so countries outside the UK. We have also begun to put it under the microscope of formal evaluation and research. ${ }^{2}$

Running a supervision quartet is not just a matter of putting four people together to discuss a case in any way they want. Unless they are very experienced and disciplined, such a conversation will easily veer off in random directions.
Instead, it helps to have some firm rules. One of these is that only one person (the case presenter) can speak about their case at a time; people cannot use the group for comparing different experiences or discussing guidelines, for example. The case presenter also needs a decent amount of time-probably a minimum of $15 \mathrm{~min}$, usually more-to talk about their case and explore the difficulties or dilemmas it raises.

The other rule is for one other specific person in the group to be nominated as the sole supervisor. This prevents everyone chipping in with comments or advice and therefore running the risk of overwhelming the case presenter with ideas. We ask the supervisor to conduct the conversation just by listening and using open questions-preferably in the least directive way possible. The overriding aim should be to allow time and space for the case presenter to reach their own conclusions by talking freely and developing new thoughts through the process of doing so.

\section{OBSERVING AND REFLECTING}

The role of the two remaining players in the supervision quartet is just as crucial. Their task is to observe the conversation as closely as they can and reflect silently on the case themselves. This will help them to pick up details, or come up with ideas and questions in their minds that the supervisor may have missed through being too closely involved in the conversation. For the same reason, it is helpful if the supervisor takes a pause every now and again to talk to the two observers about what has caught their attention, and to ask if there are questions they would pose if they were in the supervisor's place. The case presenter just listens to this reflective exchange, without taking part in it. By listening in this way, the presenter will often pick up new ideas, and develop further ideas as a result. When this reflective exchange with the observers is over, the supervisor resumes their conversation with the presenter by asking: 'Where are you now in your thoughts?'.

When a piece of supervision has progressed through two or three cycles like this-a few minutes of supervision, followed each time by a reflective breakthe presenter often gains an entirely new understanding of the case. Indeed, presenters usually say how much they appreciate hearing a range of different views in this format without having to give a response or defend their own position. At the end of the supervision some 
presenters still want to ask for direct advice from the group. However, more often than not, they feel the conversational method has itself generated enough ideas for them to know what to do next, without needing any explicit advice.

\section{LEARNING THE APPROACH}

There are variations on this basic method. When people are learning the approach for the first time, it helps to have an additional, more experienced person present as a trainer. This person-a second cello, as it were-can coach the supervisor and observers in how to craft suitable questions to ask the case presenter. The facilitator can also invite the group to 'freeze' the conversation if necessary, in order to make sure everyone is comfortable with the method, or to offer some ideas about the best way of using it. Another way of teaching it is to have a quartet carrying out supervision in a virtual 'fishbowl', with a circle of people sitting around them and watching them apply the method.

The reasons for conducting supervision in this way are both clinical and educational. The cases brought by the presenters are real, live ones, and they require genuine, workable solutions. The people participating in the quartets are clinicians who want to develop their capacity for reflective practice and mutual support. At the same time, it would be no exaggeration to say that the method also has an aesthetic dimension to it. Good medical conversations depend on sensitivity to theme tone, volume, pitch, rhythm, timing and harmony just as good musical performances do. It is possible to supervise colleagues-or indeed consult with patients-in a way that will move and inspire them, as you would by playing a string instrument with skill. In medicine, as in music, the best way of learning to excel may be... to join a quartet.

Competing interests None.

Provenance and peer review Commissioned; internally peer reviewed.

Published Online First 7 February 2012

Postgrad Med J 2012;88:185-186.

doi:10.1136/postgradmedj-2012-130793

\section{REFERENCES}

1. Launer J, Halpern H. Reflective practice and clinical supervision: an approach to promoting clinical supervision among general practitioners. Work Based Learn Prim Care 2006;4:69-72.

2. Bullock A, Monrouxe L, Atwell C. Evaluation of the London Deanery Training Course 'Supervision Skills for Clinical Teachers' (Working paper 141). Cardiff: University of Cardiff School of Social Sciences, 2011. http://www.cardiff.ac.uk/socsi/resources/wp141.pdf (accessed 14 Jan 2012). 